Haemostasis 1988;18(suppl. 1):193-196

\title{
List of Authors and Speakers
}

List of Authors and Speakers

of the

International Meeting and Discussion Symposium, Vienna July 2-4, 1987

on

Plasminogen, Function and Assay, Clinical Significance

organized by

Abt. für Klinische Haemostaseologie und Trans fusionsmedizin, Universitätskliniken

des Saarlandes*

Anderle, K.

Dr. med.

Immuno AG Industriestraße 72 A-1220 Vienna Austria

Binder, B.R.

Univ. Prof. Dr. med.

Laboratory for Clinical Exp. Physiol. Dept. of Medical Physiology University of Vienna A-1090 Vienna Austria

Castellino, FJ.

Prof, of Biochemistry Dean College of Science

Dept. of Chemistry University of Notre Dame Notre Dame Indiana 46556 U.S.A.

Ehrenreich, F.

Dr. med.

Laboratory for Clinical Exp. Physiol. Dept. of Medical Physiology University of Vienna A-1090 Vienna Austria

* under the auspices of "Österreichische Ärztekammer" (Referat für Labormedizin) and in cooperation with the "Österreichische Gesellschaft für Bioanalytik"

194

6. List of Authors and Speakers

Fröhlich, A.

Magister phil.

Immuno AG Industriestraße 72 A-1220 Vienna Austria

Gaffney, P. J.

PhD DSC MRC Path

National Institute for Biological

Standards and Control

Blanche Lane, South Mimms, Herts. 
EN63QG

United Kingdom

Hartl, D.

Dr. med.

Allgemeine Poliklinik der Stadt Wien Mariannengasse 10 A-1090 Vienna Austria

Hedner, U. Dr.

Novo Research Institute Novo Alle DK-2880 Bagsvaerd Denmark

Kaeser, A. Dr. med.

Immuno GmbH Slevogtstraße 3-5 D-6900 Heidelberg Germany (F.R.)

Kakkar, VV. FRCS FRCSE Prof.

Thrombosis Research Unit King's College School of Medicine Denmark Hill London SE5 8RX United Kingdom

Leipnitz, G.

Dr. med.

Abt. für Klinische Haemostaseologie und Transfusionsmedizin Universitätskliniken Haus 75 D6650 Homburg/Saar Germany (F.R.)

Mannhalter, Ch.

Univ. Doz. Dr. rer. nat.

First Dept. of Medicine

University of Vienna Medical School

A-1220 Vienna

$\Delta$ ne†ríc $1 / 8$

6. List of Authors and Speakers

195

Mayr, W.R.

Univ. Prof. Dr. med.

Dept. of Transfusion Medicine RWTH Aachen Pauwelstraße D-5100 Aachen Germany (F.R.)

Miyashita, C.

Dr. rer. nat.

Abt. für Klinische Haemostaseologie und Transfusionsmedizin Universitätskliniken Haus 75 D6650 Homburg/Saar Germany (F.R.)

Philapitsch, A.

Dr.

Immuno AG

Industriestraße $72 \mathrm{~A}-1220$ Vienna Austria

Scharrer, I. 
Univ. Prof. Dr. med.

Dept. of Internal Medicine University Hospital D-6000 Frankfurt/Main Germany (F.R.)

Schoppmann, A. Univ. Doz. Dr. phil.

Immuno AG

Industriestraße $72 \mathrm{~A}-1220$ Vienna Austria

Takada, A. Prof. MD.

Dept. of Physiology

Hamamatsu University

Medical School

3600 Handa-cho Hamamatsu-shi

Shizuoka-ken

Japan 431-31

Takada, J.

MD.

Dept. of Physiology

Hamamatsu University

Medical School

3600 Handa-cho Hamamatsu-shi

Shizuoka-ken

Japan 431-31

196

6. List of Authors and Speakers

Tilsner, V.

Univ. Prof. Dr. med.

Dept. of Coagulation Disorders University Clinic of Surgery and Radiology D-2000 HamburgEppendorf Germany (F.R.)

Vinazzer, $\mathrm{H}$.

Univ. Prof. Dr. med.

Blood Coagulation Laboratory

A-4020 Linz

Austria

Wenzel, E.

Univ. Prof. Dr. med.

Abt. für Klinische Haemostaseologie und Transfusionsmedizin D-6650 Homburg/Saar Germany (F.R.) 